\title{
La Révolution française dans l'espace médiatique
}

Annie Duprat, Cécile Guérin, Aurore Chery, Pascal Guimier, Pierre Serna, Roland Timsit, Emmanuel Laurentin et Emmanuel Fureix

\section{(2) OpenEdition}

\section{Journals}

Édition électronique

URL : https://journals.openedition.org/ahrf/13179

DOI : $10.4000 /$ ahrf. 13179

ISSN : 1952-403X

Éditeur :

Armand Colin, Société des études robespierristes

Édition imprimée

Date de publication : 1 juin 2014

Pagination : 135-161

ISBN : 978-2-908327-68-7

ISSN : 0003-4436

Référence électronique

Annie Duprat, Cécile Guérin, Aurore Chery, Pascal Guimier, Pierre Serna, Roland Timsit, Emmanuel Laurentin et Emmanuel Fureix, "La Révolution française dans l'espace médiatique », Annales historiques de la Révolution française [En ligne], 376 | avril-juin 2014, mis en ligne le 01 juin 2017, consulté le 01 juillet 2021. URL : http://journals.openedition.org/ahrf/13179 ; DOI : https://doi.org/ 10.4000/ahrf. 13179 
REGARDS CROISÉS

\title{
LA RÉVOLUTION FRANCGAISE DANS L'ESPACE MÉDIATIQUE
}

\author{
Annie DUPRAT \\ Cécile GUÉRIN \\ Aurore CHERY \\ Pascal GUIMIER \\ Pierre SERNA \\ Roland TIMSIT \\ Emmanuel LAURENTIN \\ Emmanuel FUREIX
}

\section{Origines du projet}

Lorsque survient en Tunisie au début de l'année 2011 une révolte brutale et profonde contre les pouvoirs en place, révolte qui provoque la chute du gouvernement Ben Ali, la tentation a été forte chez les intellectuels français, et, singulièrement, parmi les historiens de la Révolution française, d'observer les événements en cours avec les lunettes de leur savoir académique et de leurs convictions de citoyens. L'un des premiers à écrire sur ce sujet a été Jean Tulard, le 18 janvier 2011, dans le quotidien Le Monde. Le 12 février suivant, Jean-Clément Martin, ancien directeur de l'IHRF, publie, également dans Le Monde, une lettre dont le titre («Le renvoi à 1789 égare plus qu'il n'éclaire ») autant que le contenu, ont suscité des réactions immédiates, chacun souhaitant défendre un avis personnel sur la question ${ }^{1}$. Quelques jours plus tard, Pierre Serna, directeur actuel de l'IHRF, poste à son tour une lettre sur le site de l'Institut. Chacun à sa manière tente de comprendre l'actualité du moment, soit en mettant l'accent sur les proximités entre le combat pour la reconnaissance des Droits de l'Homme en France au XVIII ${ }^{\mathrm{e}}$ siècle et les revendications tunisiennes, soit

(1) Le titre est de l'auteur qui avait également proposé à la rédaction du Monde «Attention, une Révolution ne cache pas toujours une Révolution ». 
en insistant sur l'identité d'insurrections se déroulant dans un contexte culturel spécifique. Ce bref débat entre l'ancien et le nouveau directeur de l'IHRF a été suivi quelques semaines plus tard par une intervention de Bernard Gainot déposée sur le même site de l'IHRF². Les tribunes médiatiques les plus diverses (radios, télévisions, sites Internet) ont vu fleurir nombre de commentaires pratiquant souvent l'analogie ${ }^{3}$. En ces temps obscurs où se joue la fin du monde de l'après seconde guerre mondiale, la tentation est grande de se réfugier derrière des références historiques, pas toujours appropriées, pour comprendre le présent et, peut-être, éclairer les enjeux de l'avenir...

Les références à la Révolution française sont perpétuellement présentes, qu'il s'agisse de la célébrer ou de la dénigrer car elle est, et demeure, un marqueur idéologique puissant. Sujet de nombreux livres, bandes dessinées, films et téléfilms car elle est le moment du bouleversement des destins individuels, objet de l'attention des hommes politiques, des leaders syndicalistes ou de tous ceux qui, à des titres divers, font profession de penser le monde et son histoire, elle a connu, par la grâce de la campagne électorale pour l'élection du président de la République en 2012, une présence nouvelle dans ce qu'il est convenu d'appeler "l'espace médiatique ». Par ce terme, nous entendons cet air du temps façonné par les réseaux sociaux, nouvel amplificateur des informations et des opinions. Des pièces de théâtre, des films, des ventes aux enchères d'objets concernant la Révolution française peuvent se dérouler mais, s'il n'y a pas de relais médiatique, l'événement sera considéré comme n'ayant pas eu lieu. Chacun d'entre nous, lecteurs des Annales historiques de la Révolution française, repérons vite des informations, des publicités, des mots ou des signes faisant référence aux individus et aux événements de la Révolution française. Mais qu'en est-il des autres ? Qu'en est-il de tous ceux qui, d'un niveau d'éducation élevé, ne font pas profession d'étudier cette période de l'Histoire mais sont très attentifs à cet air du temps diffusé par l'espace médiatique?

Pour organiser cette rubrique de « Regards croisés », nous avons contacté des personnes de profession et d'âge différents. Nous leur avons posé au départ des questions identiques afin de les faire converser à distance. En effet, pour des raisons évidentes de disponibilité, il n'a pas

(2) http://ihrf.univ-paris1.fr/

(3) Le site http://revolution-francaise.net a même repris un article publié dans le journal Libération le 15 janvier 2011 portant le titre « Aujourd'hui, on a pris la Bastille » par Léa-Lisa Westerhoff... 
été matériellement possible de les réunir au même moment en un lieu unique. Par mon questionnement, je suis en quelque sorte devenue le lieu en question. Mais, les réponses étant très différentes selon les cas, j'ai pu approfondir (ou non) la discussion et aller plus loin dans la recherche d'informations. Enfin, on ne trouvera pas ci-dessous de grandes notions conceptuelles ou historiographiques mais seulement quelques jalons pour la réflexion. Le sujet de ce «Regards croisés » m'est venu alors que j'avais le sentiment de rencontrer des références révolutionnaires presque à chaque pas. Je me suis demandé alors si d'autres personnes, dans et hors de la sphère universitaire, partageaient, ou non, mon opinion.

\section{Choix des participants}

Le cadre chronologique du questionnement a porté sur trois années calendaires, 2011, 2012 et 2013. J'ai contacté deux étudiantes confirmées, Cécile Guérin et Aurore Chéry, deux journalistes, Emmanuel Laurentin et Pascal Guimier, un metteur en scène de théâtre, Roland Timsit et deux universitaires, Emmanuel Fureix et Pierre Serna. Globalement, je les ai interrogés d'abord sur leurs manières de s'informer et leur appétence plus ou moins forte pour Internet et les réseaux sociaux afin d'éviter les biais dans leurs réponses.

\section{Comment vous informez-vous?}

Cécile Guérin est une jeune normalienne qui prépare l'agrégation d'Anglais à l'ENS Lyon. Lorsque je l'ai rencontrée, elle bouclait un stage d'édition dans la revue Transfuge.

\section{Cécile Guérin}

J'écoute peu la radio française, j'écoute en revanche presque quotidiennement des chaînes en anglais, je ne regarde pas la télévision mais je suis très informée par mes réseaux sur mon compte Facebook. J'achète assez souvent un quotidien, rarement des magazines, hebdomadaires ou mensuels à l'exception du Monde diplomatique, d'une part parce qu'il offre un regard différent sur l'actualité, et des articles documentés et de qualité, et d'autre part parce que j'en apprécie la ligne éditoriale. Je m'intéresse personnellement aux minorités noires, en Grande-Bretagne et aux États-Unis. Je lis beaucoup, surtout des romans anglo-saxons ou des livres 
d'études portant sur mes sujets de prédilection. Je vais au cinéma, mais jamais pour y voir des films historiques.

Aurore Chéry est doctorante en histoire moderne à l'Université Lyon III (sujet de la thèse : "L'image du roi en France sous Louis XV et Louis XVI », sous la direction de Bernard Hours). Je l'ai croisée en novembre 2012 lors d'un colloque à Lyon, organisé par nos collègues Paul Chopelin et Sylvène Edouard.

\section{Aurore Chéry}

Je m'informe beaucoup via Internet (sites divers, blogs et réseaux sociaux). En tant que membre du CVUH, je m'intéresse de très près aux usages publics de l'histoire. Je regarde peu la télévision ; je suis passionnée par le théâtre et j'y vais le plus souvent possible. » Aurore Chéry est co-auteur avec Christophe Naudin et William Blanc du livre Les historiens de garde. De Lorant Deutsch à Patrick Buisson, la résurgence du roman national, Paris, Éditions Inculte, 2013.

J'ai fait la connaissance de Roland Timsit, homme de théâtre, après la représentation de la pièce d'André Bénédetto, Thermidor-Terminus au Grand Orient de France en septembre 2013. Homme du livre, il s'informe aussi parfois par la presse, sans y attacher grande attention.

Emmanuel Laurentin a reçu une double formation, historien et journaliste. Producteur et animateur de l'émission quotidienne La Fabrique de l'Histoire, il est, par attirance personnelle et par nécessité professionnelle, un homme de médias et de réseaux.

\section{Emmanuel Laurentin}

Je reçois les dépêches d'agence, je lis les messages Facebook et Twitter mais aussi la presse écrite, quotidienne ou les magazines. J'ai la possibilité de lire de nombreux sites dont la richesse est étonnante et permet d'apprendre les informations nouvelles, comme les découvertes archéologiques, ou de comprendre les renouvellements historiographiques. Je fais le tri moi-même afin de définir les sujets de mes émissions, qui seront traités par les membres de mon équipe ou par moi-même, en fonction de nos envies. J'essaie d'aller souvent au théâtre, plus rarement au cinéma.

Pascal Guimier a une double formation de germaniste et de journaliste. J'ai fait sa connaissance grâce à un échange de courriers électroniques 
portant sur le livre Révolutions et mythes identitaires que j'ai dirigé en 2009 chez Nouveau Monde. Après avoir travaillé plusieurs années pour France 2 et Arte, il est à présent directeur de la rédaction de TV5 Monde.

\section{Pascal Guimier}

Je suis informé par les dépêches d'agence et par toutes les ressources électroniques de ma profession. Je lis beaucoup, des romans, des essais, des biographies ; je vais volontiers au cinéma, j'aime bien les films « en costumes » mais je ne vais guère au théâtre.

Emmanuel Fureix est un ami personnel de longue date. Actuellement maître de conférences en histoire contemporaine à l'UPEC (Créteil), il est membre de l'IUF et secrétaire de rédaction de la Revue d'histoire du XIX ${ }^{e}$ siècle.

\section{Emmanuel Fureix}

Je suis informé par la radio (de France-inter à RFI en passant par France-culture), un peu par la télévision et par une multitude de sites Internet et de blogs, mais pas par les réseaux sociaux. Je contribue à quelques blogs comme « La Vie des Idées » pour la critique de livres et je suis l'actualité, en consultant fréquemment le site H-France. Je fais partie du comité de rédaction de la revue $R H$ 19. Je vais volontiers au cinéma. Mon regard sur la Révolution est un peu décalé par mon intérêt de dix-neuvièmiste.

Pierre Serna a été interrogé en tant qu'actuel directeur de l'IHRF. Je m'informe par la lecture d'un quotidien, Le Monde, et par le mail mais ni par les réseaux sociaux ni par les blogs. Le site de l'IHRF http://ihrf.univ-paris1.fr/ - met en ligne des informations que j'ai validées ainsi que des liens choisis sur les sites revolution-francaise.net/ou le site de la Société des études robespierristes (http://ser.hypotheses.org/). Je lis beaucoup, des livres d'histoire, mais aussi des essais et des bandes dessinées historiques ${ }^{4}$. J'aime beaucoup le cinéma, les films historiques mais aussi les films d'anticipation qui racontent beaucoup sur notre société, comme La planète des singes, que l'on peut lire comme une métaphore de société en train de se constituer et la tension entre l'Égalité revendiquée et les

(4) Pierre Serna est membre du jury du prix de la bande dessinée historique qui se tient chaque année lors du festival de l'Histoire de Blois et qui est présidé par Pascal Ory. 
tentatives de domination par quelques-uns. Je vais également au théâtre aussi souvent que possible.

\section{Avez-vous perçu récemment un retour des références sur la Révolution française ?}

\section{Cécile Guérin}

Oui, lors d'un meeting de Jean-Luc Mélenchon, j'ai vu beaucoup de bonnets phrygiens ! J'ai suivi la grande manifestation du 28 mars 2012, annoncée comme une nouvelle prise de la Bastille. Il me semble que pour beaucoup de jeunes gens de ma génération (j'extrapole sans doute un peu), la Révolution française est souvent «connue » non pas par la lecture de revues de spécialistes, mais par le roman, le cinéma, la culture populaire... etc. Mises à part les polémiques occasionnelles et certaines commémorations qui peuvent conduire à une plus grande visibilité de la Révolution dans les médias (et encore : je n'étais pas née au moment du bicentenaire de la Révolution ; cela parle donc vraisemblablement peu aux gens de mon âge). C'est souvent grâce au cinéma qu'un jeune non-historien entend parler de la Révolution hors de sa scolarité au lycée. Je n'ai pas $\mathrm{vu}$ «Les Amants de la Bastille» parce que je ne suis pas une grande amatrice de comédies musicales mais, si je l'étais, peut-être y serais-je allée et je connais un certain nombre de personnes qui se sont déplacées pour l'occasion. Autre fait complètement anecdotique : en 2012/2013, j'ai été assistante de français dans une université anglaise et mes étudiants britanniques, qui avaient la vingtaine, ont souvent exprimé l'idée que, de leur point de vue, la Révolution française était d'actualité. Ils se fondaient pour dire cela sur la sortie et le succès de la comédie musicale Les Misérables. En allant voir Les Misérables, ils pensaient en effet regarder un film sur la Révolution de 1789. Mais ça, c'est un autre problème... Finalement il me semble que l'idée de révolution revient de manière intermittente dans les médias mais que la Révolution française reste un domaine de spécialistes, peu audible dans l'espace public, ce qui est bien dommage d'ailleurs. Mais c'est une perception tout à fait subjective bien sûr.

Aurore Chéry transpose la question sur le plan de la patrimonialisation de la mémoire. 
Je ressens pour ma part une saturation mémorielle, ce qui me semble dommageable parce qu'elle occulte la dimension politique des événements en cours.

Roland Timsit, Pascal Guimier et Emmanuel Laurentin n'ont pas remarqué de retour particulier de la Révolution française dans l'espace médiatique, hormis dans le verbe de Mélenchon. Comme je fais remarquer à Emmanuel Laurentin qu'étant donné ses fonctions « d'instituteur national radiophonique », formule dont il s'est défendu mais qui l'a fait sourire, il pourrait sans doute développer davantage.

\section{Emmanuel Laurentin}

Après le Bicentenaire, il y a eu une diète et sans doute est-ce pour cela que la réapparition de la Révolution française dans l'espace médiatique paraît surprenante. Depuis les publications de Furet, il y a eu renouvellement des positions historiographiques dans plusieurs directions, mais en particulier concernant la Terreur ou la Vendée 5 .

Questionné sur la présence de la Révolution dans La Fabrique de l'Histoire :

On peut grosso modo l'évaluer à environ 4 semaines sur un total de 120 semaines d'émissions, auxquelles il faudrait ajouter la présentation de l'actualité dans les émissions du vendredi. Le renouvellement historiographique ne s'est réellement produit que depuis le début des années 2000 .

Questionné à son tour sur un éventuel retour des références révolutionnaires dans l'espace public, Emmanuel Fureix remarque à la fois une multiplication et un affadissement de ces références par des emprunts tronqués et paradoxaux.

\section{Emmanuel Fureix}

Le Point, peu suspect de sympathies ultrarévolutionnaires, a fait sa une sur un éventuel retour d'un 1789 antifiscal. Le site Atlantico a évoqué un nouveau 1788. Des opposantes au «mariage pour tous », tout droit venues de l'ouest parisien, ont osé manifester déguisées en femmes sans-culottes ou en Mariannes, arborant cocardes et bonnets phrygiens.

(5) Ce point sera développé plus loin. 
Quant à Marine Le Pen, dans son discours près de la statue de Jeanne d'Arc, le $1^{\text {er }}$ mai 2011, elle a fait référence à Robespierre mais sans citer son nom ! Pourtant, dans le texte distribué à la presse auparavant, le nom du Conventionnel figurait bel et bien. Voici ce qu'a dit Marine Le Pen :

« Un grand révolutionnaire l'avait théorisé en son temps : « L'homme de génie qui révèle de grandes vérités à ses semblables est celui qui a devancé l'opinion de son siècle. La nouveauté hardie de ses conceptions effarouche toujours leurs faiblesses et leur ignorance. Toujours les préjugés se ligueront avec l'envie pour le peindre sous des traits odieux ou ridicules $»^{6}$.

Par ailleurs, lors des «printemps arabes », les commentateurs ont fréquemment fait référence aux révolutions du $\mathrm{XIX}^{\mathrm{e}}$ siècle, surtout à 1848 , avec des lunettes « contagionnistes », sans toujours respecter ce qui surgissait de nouveau et d'original dans le présent. Dans ces propos, il faut surtout lire en creux une surprise face à l'opinion communément admise de la fin de 1989.

Lorsque j'ai posé la question de l'irruption éventuelle des références à la Révolution Française, Pierre Serna m'a répondu qu'étant donné sa position institutionnelle, il n'avait pas cessé de la rencontrer...

\section{Quelles pièces de théâtre avez-vous vu récemment ?}

J'ai orienté le questionnement en citant quelques titres : Notre Terreur [création collective mise en scène par Sylvain Creuzevault], Olympe de Gouges [Elsa Solal], La mort de Danton [Büchner], Charlotte Corday [Benoît Lepecq] et Thermidor-Terminus ou la mort de Robespierre [André Bénédetto].

Roland Timsit explique pourquoi il a monté cette pièce pour le festival d'Avignon 2013.

La pièce a été créée en 1989 à la Cartoucherie dans une mise en scène d'Ariane Mnouchkine. Après la mort, en 2009, de Bénédetto avec qui j'avais déjà eu l'occasion de travailler, plusieurs hommages lui ont été rendus. J'ai décidé de procéder à un toilettage de la pièce, en la réduisant. C'est une pièce politique, ni militante ni engagée mais profondément

(6) On sait que Marine Le Pen pratique souvent les références à l'Histoire : elle y a fait aussi l'apologie de « la Grande Nation »... 
humaine. Elle met en scène deux personnages seulement, en prison, juste après Thermidor : Duplay et Buonarotti. Duplay, le logeur et ami de Robespierre, ne désire que le silence et, dans un premier temps, refuse d'argumenter face aux critiques de Buonarotti qui veut comprendre et lui reproche l'échec de la Révolution. Ce qui m'a intéressé ici, au-delà des deux personnages, c'est le souffle de l'époque, le souffle des événements qui contraint les individus. Je n'ai pas revu la récemment la très bonne pièce Charlotte Corday, de Benoît Lepecq, qui avait été astucieusement montée par la Compagnie du Chat de Chéschire. Comme Thermidor-Terminus, elle met en scène deux personnages seulement, Charlotte Corday et FouquierTinville. Les répliques montrent une Charlotte Corday beaucoup plus dure, presque fanatique, que Fouquier-Tinville lui-même. En revanche, je n'ai pas beaucoup d'affinités avec le travail d'Annie Vergne et de Clarissa Palmer pour Olympe de Gouges.

\section{Aurore Chéry}

Je vais voir des pièces de théâtre très diverses, ne portant pas particulièrement sur la Révolution française mais je garde un souvenir puissant de La mort de Danton, de Büchner, vu il y a quelques années au théâtre de l'Odéon. C'était la version de Lavaudant qui a d'ailleurs été rejouée à la MC 93 il y a peu. J'avais aussi vu une version à Vienne en allemand, en 2001. Je suis particulièrement intéressée par le travail des metteurs en scène ; je vais régulièrement à la Maison de la Culture du 93.

Mais Aurore Chéry n'a pas eu connaissance des pièces ni sur Charlotte Corday ni sur Olympe de Gouges.

J'ai un souvenir très fort de la pièce Notre Terreur que j'ai vue quand elle tournait dans les Yvelines et j' ai assisté au débat qui avait été organisé au théâtre de la Colline. Pour moi, c'était une vraie réussite, j' aime beaucoup les pièces collectives qui partent d'improvisations et ça ne me semble pas être du même ordre que les pièces sur Corday ou Olympe de Gouges, plus classiques ou Thermidor-Terminus. Mais si l'image de Charlotte Corday tend à l'emporter de nos jours sur celle d'Olympe de Gouges, les publics sont différents.

Pierre Serna en revanche a vu plusieurs de ces pièces et a d'ailleurs été convié à animer un débat à la suite de Notre Terreur au théâtre de la Colline à Paris. 


\section{Pierre Serna}

J'ai senti une grande attention chez les spectateurs. Le débat qui a suivi, avec Sophie Wahnich et Alain Badiou a été d'une grande intensité (sourire). Le débat, tendu, portait sur la nécessité d'une politique de guerre, remarquablement bien vue par le jeu des acteurs selon moi, et les impératifs de la construction morale de la République. J'ai été fasciné par l'ampleur du travail et ce que je crois être la justesse de leurs intuitions autour de la fameuse table verte du Comité de salut public. J'ai pu parler avec « Barère » ensuite, et de la difficulté à cerner le personnage. Bien sûr, j'ai vu avec toujours autant de plaisir La mort de Danton de Büchner mais je n'ai pas vu ni Corday ${ }^{7}$, ni Olympe de Gouges, ni Thermidor.

Bien que grand amateur de théâtre, Emmanuel Laurentin n'a vu qu'une seule des pièces mentionnées ici, Notre Terreur.

\section{Emmanuel Laurentin}

J'ai été très impressionné par la force des acteurs et de ce texte qui est en grande partie issu de l'improvisation. On sent le souffle révolutionnaire, le souffle de la réappropriation d'une Histoire vieille de plus de deux siècles par des jeunes comédiens vivant au $\mathrm{XXI}^{\mathrm{e}}$ siècle. L'histoire de la Révolution française est devenue ici la matrice d'un engagement politique. Mon précédent grand souvenir de théâtre, La mort de Danton, était très culturel puisque la pièce de Büchner (1935) était déjà entrée dans un patrimoine reconnu et célébré.

\section{Quels films ou téléfilms portant sur la Révolution française avez-vous vu récemment ?}

Dans ce registre d'un art très populaire, $j$ ' ai eu la surprise de constater que la plupart de mes interlocuteurs ignoraient les titres que je leur ai proposés (titres peu nombreux étant donné le cadre chronologique restreint de l'enquête : Les adieux à la reine et Royal affair). Bien qu' amateurs de cinéma, Pascal Guimier et Emmanuel Fureix n'ont vu aucun des films récents portant sur la Révolution française. Mais il est des films en quelque sorte « patrimoniaux » qui ont marqué leur mémoire, comme le Danton de Wajda pour Emmanuel Laurentin et Emmanuel Fureix ou encore L'Anglaise et le duc d'Eric Rohmer pour Pascal Guimier et Emmanuel

(7) Raymonde Monnier avait mentionné le Charlotte Corday à la Cartoucherie de Vincennes dans un mail circulaire adressé aux membres du conseil d'administration de la SER. 
Fureix qui confie « en être sorti à la fois impressionné et irrité par le regard porté sur la foule et la violence révolutionnaires ».

\section{Aurore Chéry}

Je suis volontiers les metteurs en scène, au cinéma comme au théâtre. Quant aux films historiques, je m'y intéresse aussi mais c'est une partie de mon travail. J'ai réfléchi sur la question des films et téléfilms (j'ai même fait un article sur le sujet pour le site de l'IHRF) mais pour moi c'est du travail et c'est vrai que je vais rarement en voir pour mes loisirs ${ }^{8}$. Je ne suis pas allée voir Les adieux à la reine au cinéma car je n'apprécie pas particulièrement le regard souvent très masculin que Benoît Jacquot porte sur les femmes. J'avais bien aimé le Marie-Antoinette de Sofia Coppola qui, au fond, me semblait bien rendre compte de ce que pouvait être Marie-Antoinette en esprit. Le film Royal Affair a été un film important pour moi. J'avais été invitée à l'avant-première et j'en attendais beaucoup mais j'ai été déçue. Je connaissais l'histoire de Caroline-Mathilde auparavant et j'ai trouvé embarrassante la manière dont était traité son personnage. Elle apparaît comme révélée à elle-même par son histoire avec Struensee, mais c'était une femme de caractère bien avant. Par exemple, elle se fait représenter en habit d'homme bien avant Marie-Antoinette. Pour moi elle est, avec Isabelle de Bourbon-Parme, l'une des princesses les plus intéressantes et les plus intelligentes de la période et là, le scénario fait un peu bluette. J'ai été très séduite par l'interprétation de tous les acteurs mais en revanche les décors et les costumes n'étaient souvent pas à la hauteur et anachroniques, ça cassait un peu le charme.

Pierre Serna fait des choix personnels.

J'ai acheté récemment l'œuvre d'Albert Capellani éditée en quatre DVD par la Fondation Jérôme Seydoux pour Pathé. Il s'agit d'une œuvre importante, des films muets réalisés entre 1913 et 1920, avec Le chevalier de Maison-Rouge - 1914 - et Quatre-vingt-treize - 1913-1921. Encore fautil voir ailleurs la Révolution. Prenons la comédie ironique Red, interprétée entre autres par le génial Malkovitch. A priori rien à voir entre cette production hollywoodienne kitch et la révolution. Regardons mieux : des retraités en pleine forme se mettent en colère et décident d'imposer leur loi. Je pense que la révolution par définition éclate toujours là où les

(8) Aurore CHÉRY, https://www.academia.edu/194294/LEvasion de louis XVI une lecon dhistoire paradoxale http://h-france.net/fffh/the-buzz/1242 
spécialistes de son histoire ne l'ont pas prévue, comme en Tunisie. Pour faire une révolution il faut être d'abord en forme physique ; ensuite en avoir le temps et n'avoir plus ou moins rien à perdre et, si possible, un projet de société. Quel groupe parmi nous peut réunir ces quelques qualités, si ce n'est le groupe «inventé » des retraités de 60 à 80 ans hyperactifs, ayant du temps, très bien formés et qui pourraient venir à se rebeller, si qui sait, la faillite d'un État rendait le versement de leur pension impossible ? Que se passerait-il ? Des vieux révolutionnaires, pourquoi pas ? Ma génération aura sa retraite vers 75 ans, exténuée... Les révolutions pourraient être faites à l'avenir dans nos pays riches mais subitement appauvris par des femmes et des hommes d'âge mûr. Cela va à l'encontre de tout enthousiasme romantico-insurrectionnel et doit donner matière à réflexion. Bien évidemment la colère est tellement palpable qu'elle peut éclater ailleurs et sous d'autres formes.

Enfin, Pierre Serna, dont on a évoqué précédemment la lecture personnelle de la série de films La planète des singes, explique l'importance qu'il attache au film danois Royal Affair (2012) de Nicolaj Arcel.

C'est un film très important à plusieurs titres. D'abord, c'est le marqueur de l'identité danoise car cette affaire d'adultère de la reine du Danemark Caroline Mathilde de Hanovre avec le médecin et homme des Lumières Johann Friedrich Struensee pose de nombreuses questions. L'une concerne le roi Christian VII, un peu débile et très caractériel, et sa façon de gouverner, tantôt comme une marionnette aux mains des grands, tantôt comme un tyran imprévisible, tantôt enfin comme un homme sous l'influence de Struensee. On voit ici se poser la question de l'incapacité du roi à gouverner, ce qui renvoie à une angoisse constante des peuples parce qu'il n'est pas possible de s'en débarrasser et que l'on ne peut pas savoir quel est l'entourage proche qui l'influence ou même gouverne le pays. On voit aussi l'attention particulière du siècle des Lumières au sort et à la liberté des femmes, même reines. L'autre concerne la figure de Struensee, venu de Prusse à la demande de Christian VII, et qui va rapidement tenter d'imposer des réformes au Danemark. Il lutte contre la misère, obtient l'abolition du servage, de la torture et d'autres réformes inspirées par la philosophie des Lumières. Mais une fois la liaison dévoilée, Struensee est arrêté et exécuté (en 1772), avec force tortures qui rappellent celles subies par Damiens en France (exécuté en 1757). Lors des promenades de la reine avec Struensee dans la campagne, on voit non seulement la misère des paysans mais aussi les mauvais traitements dont ils sont victimes de la part de leurs seigneurs - jusqu'à la scène où le cadavre d'un homme battu à 
mort est ligoté sur une barrière et sa veuve ne peut pas s'en approcher... La misère dans les rues de Copenhague est également montrée ! Une reine adultère, un roi incapable, un peuple souffrant : ainsi, dans les années 1770 en Europe du nord peut-on observer quelques-uns des caractéristiques de la France des années 1780.

\section{Quelles autres sources d'information consultez-vous ?}

J'ai orienté le questionnement sur les téléfilms, les romans et la bande dessinée. La BD n'a pas été souvent citée, sauf de la part de Pierre Serna, membre du jury de la BD historique de Blois.

\section{Pierre Serna}

J'ai participé à un long débat avec Jean-Louis Bocquet, auteur d'une monumentale bande dessinée Olympe de Gouges, avec Catel qui je crois a eu un vrai succès mérité de librairie [parution mars 2012]. J'ai été impressionné par le travail préparatoire et l'émotion qui se dégage dans le long portrait d'Olympes en nuances, sans parti pris. Qu'il me soit permis de citer le très beau travail de Bourgeon La petite fille Bois Caïman I et II, (12 bis éd.) qui place au cœur de l'intrigue la révolte de Saint Domingue, et de Yslaire et Carrière Le ciel au-dessus du Louvre, (Futuropolis/musée du Louvre éd.) interprétation libre et sombre mais très suggestive et belle d'un tableau de David peint pendant la Terreur.

Interrogé sur les bandes dessinées, Pascal Guimier répond immédiatement.

J'ai beaucoup apprécié la bande dessinée sur Olympe de Gouges, très bien réalisée, très informée et tout à fait passionnante !

Il n'y a pas eu d'actualité particulièrement remarquée en matière de romans ayant pour objet, ou simplement pour toile de fond, la Révolution française durant les trois années considérées. Des revues et des magazines ont pu consacrer des numéros spéciaux à notre sujet à l'instar d'autres périodes de l'Histoire de France.

\section{Pierre Serna}

Il y a eu une actualité des magazines. Citons le numéro 777 de la revue Historia par exemple, paru en septembre 2011 sous le titre Robespierre, le psychopathe légaliste, dont la publication a fait des vagues dans le milieu des historiens de la Révolution française. Il y a eu un bilan historiographique proposé par Les collections de l'Histoire, $\mathrm{n}^{\circ}$ 60, juillet-août 2013, auquel 
ont participé plusieurs membres de l'IHRF dont Virginie Martin, Guillaume Mazeau et moi-même.

Je rappelle pour mémoire la réflexion plus généraliste et très illustrée dans le magazine GéoHistoire qui a consacré son numéro spécial d'été sous le titre 1789-1795 La Révolution française et plusieurs expositions dont Culture populaire et Révolution française (XXe et XXI siècles) au Musée de la Révolution française à Vizille (28 juin 2013-28 avril 2014).

On peut aussi la repérer dans la presse institutionnelle de la Sorbonne car le premier numéro de la revue Panthéon-Sorbonne Magazine (janvierfévrier 2013) a été publié sous le titre « Sommes-nous en 1788 ? »; on y trouve un article de Pierre Serna, écrit en tant que directeur de l'IHRF, « Nous sommes en 1788... » [p. 12-15]. En me présentant ce numéro, notre collègue développe.

\section{Pierre Serna}

La couverture figure un masque d'Anonymous, qui appartient à la culture underground de la bande dessinée américaine $V$ pour Vendetta d'Alan Moore et David Lloyd (1980). Le héros porte des vêtements sombres, un masque à la Guy Fawkes, l'un des membres de la conspiration des poudres qui a fomenté la tentative d'attentat contre le Parlement d'Angleterre le 5 novembre 1605. Il est intéressant de voir que le choix de la couverture d'un numéro ayant pour titre « Sommes-nous en 1788 ? » se soit porté sur cette image, bien dans la contre-culture du XXI ${ }^{\mathrm{e}}$ siècle, car je ne sais si tous ceux qui se réfèrent à ce symbole connaissent exactement la conspiration des poudres, ce qui par ailleurs n'enlève rien à leur légitime colère !

Enfin, notons que le choix retenu pour thème de la semaine de l'Histoire organisée conjointement par l'ENS-Ulm et par le CFJ (Centre de Formation des Journalistes auquel Pascal Guimier apporte son expertise de professionnel) a été « Histoires de révolutions ». Certes, les étudiantsjournalistes avaient dans la tête les révolutions arabes mais ont fait une place non négligeable à la Révolution française et aux révolutions du $\mathrm{XIX}^{\mathrm{e}}$ siècle.

Concernant les téléfilms et répondant à une de mes questions sur la façon dont les médias exploitent et le plus souvent trahissent les universitaires qu'ils contactent volontiers pour leur expertise gracieuse, Pierre Serna a une réponse positive : 


\section{Pierre Serna}

Je suis sollicité, en tant que directeur de l'Institut. Mais je ne réponds favorablement que lorsque deux critères sont réunis : que ma participation serve l'Institut et que les sollicitations viennent de personnes sérieuses. Je tiens à ce qu'il y ait un dialogue en direction des enseignants du secondaire. D'ailleurs, le site de l'IHRF a reçu un label de qualité de la part du ministère de l'Éducation nationale, en raison de la variété et de l'aspect pluriel des informations qu'il délivre.

À une question portant sur les téléfilms auxquels il a participé, Pierre Serna répond :

J'ai de très bons souvenirs du tournage de trois émissions, l'une portant sur les insurrections parisiennes au fil de l'Histoire, pour la chaîne parlementaire je crois, l'autre sur les trésors des Archives nationales présentée en début d'année sur la chaîne France 5, enfin, un téléfilm francoallemand produit par Arte sur les significations du 14 juillet. L'équipe était respectueuse et il n'y a pas eu aucune manipulation de mes propos. Je regrette cependant que l'argumentaire des auteurs du scénario en reste souvent à une histoire événementielle des individus connus ou à celle de quelques faits marquants. Il y aurait tant à dire sur les conditions de vie à Paris, une capitale empestée par les odeurs des fabriques de suif (pour savon, pour bougies etc.) qui étaient implantés faubourg Saint-Antoine mais aussi dans la plaine de Vaugirard. Les descriptions de l'époque montrent une ville empuantie, toujours recouverte d'un épais nuage de suie. Les incendies étaient fréquents et dangereux - on se souvient du plus célèbre d'entre eux, celui des établissements Réveillon en avril 1789. La ville est en permanence sous cette menace diffuse dans l'air. D'ailleurs, sous l'Ancien Régime, les administrateurs de l'hygiène ne cessent de faire des rapports et de dresser des procès-verbaux ${ }^{9}$. Donc, il est vraiment dommage que cette dimension des conditions d'existence des Parisiens ne soit jamais prise en compte dans les téléfilms.

Le sentiment d'Emmanuel Fureix concernant ces interventions médiatiques est réservé :

J'ai été contacté, via l'entremise de Sophie Wahnich, pour participer à un projet original proposé par le réalisateur Pierre-Oscar Lévy. Il s'agissait d'un documentaire sur l'histoire des révolutions et des insurrections à Paris depuis 1789. Le parti-pris était de mettre en valeur les lieux de surgissement

(9) Momcilo MARKOVICS, « La Révolution aux barrières : l'incendie des barrières de l'octroi à Paris en juillet $1789 », A H R F, \mathrm{n}^{\circ} 372,2013 / 2$, p. 27-48. 
d'événements révolutionnaires ou de leur mémoire - le cimetière du PèreLachaise, Montmartre, la place de la Bastille etc. Nous nous sommes déplacés dans ces différents lieux ; au passage, j'ai eu ainsi l'opportunité de monter au sommet de la colonne de Juillet et de visiter les caveaux des révolutionnaires de 1830 et 1848. Entre la préparation documentaire et les déplacements, j'ai été requis durant une bonne semaine. Or, des mois plus tard, aucun des historiens ayant participé à ce projet n'a eu de nouvelle du devenir de ce travail. Pareille désinvolture est grave car les promoteurs et réalisateurs de documentaires et de films, qui ont besoin de nos compétences scientifiques - toujours dispensées gratuitement d'ailleurs - manquent souvent de la plus élémentaire courtoisie. Plus généralement certains documentaires souffrent d'une absence de maturation, le temps de la prise de vues étant aussi celui de la conception intellectuelle, donc un peu tardive...

\section{Aurore Chéry}

J'avais été contactée pour participer au documentaire "Tête-à-tête avec Louis XVI" de Frédéric Compain en 2011. Cela avait été pour moi une excellente expérience parce que Frédéric est un véritable réalisateur de documentaires. J'entends par-là que, même lorsqu'il travaille pour la télévision, il ne se contraint pas à un cahier des charges particulier, il privilégie toujours son regard singulier de réalisateur. Cela lui demande souvent de se battre contre les programmateurs des diverses chaînes. Il en paie aussi régulièrement le prix en se voyant reléguer à des horaires de diffusion impossibles mais cela en vaut la peine. Quand il travaille avec des historiens qui ont un véritable souci de popularisation de l'histoire, qui sont prêts à faire des efforts pour expliquer les points complexes aux nonspécialistes, il est à leur écoute, sait mettre leur parole en valeur. Je crois que l'historien a besoin de ce type d'échanges pour pouvoir pleinement remplir son rôle social et retrouver le chemin du public au-delà de la seule recherche évaluée par ses pairs.

\section{Historiographie et controverses}

Cécile Guérin, qui se souvient assez bien de ses cours de lycée - enseignement secondaire et classes préparatoires - sait qu'il y a deux théories explicatives de l'histoire de la Révolution française, celle de Soboul et celle de Furet. L'histoire conduite par Furet et ses disciples est une histoire conservatrice, face à l'histoire marxiste de Soboul. Instinctivement, 
je peux citer les grandes figures de la Révolution que sont Danton et Robespierre, mais pas grand-chose d'autre. Je n'ai pas entendu parler de la vente aux enchères des papiers de Robespierre [18 mai 2011], ni de l'hommage à la Convention organisé par la Société des études robespierristes [21 septembre 2012]. Quant à l'affaire de la reconstitution du visage de Robespierre [12 décembre 2013] — j'en ai entendu parler, mais une seule fois seulement : j'ai lu un article à ce sujet dans le journal britannique The Guardian (je ne saurais pas vous donner la date précise, probablement fin 2013), bizarrement je n'en ai pas entendu parler à la radio française ni dans la presse mais peut-être n'ai-je pas lu attentivement l'actualité à ce moment-là. Dans l'ensemble, mais là encore ayant un regard extérieur et n'étant pas étudiante en Histoire, je n'ai pas l'impression que l'événement ait pris une dimension plus importante depuis la fin de l'année dernière. Il me semble que le fait de reconstituer les visages des personnages historiques est une tendance assez récente (dans mes souvenirs, cela a également été fait pour Henri IV, non ?). Peut-être que cela participe d'une volonté d'humanisation ou d'un intérêt grandissant pour la « vie » des figures historiques aux dépens de l'action politique, je ne sais pas. À titre personnel, ce n'est pas le genre de découverte ou d'entreprise qui me pousse à m'intéresser davantage à l'histoire. J'aurais davantage tendance à m'intéresser à la Révolution à l'occasion d'une commémoration, ou si un livre propose une approche un peu différente de la Révolution, par exemple en s'intéressant à une catégorie de la population pendant les événements, ou en prenant appui sur les discours des acteurs politiques de l'époque. Le fait de savoir que Robespierre avait le visage déformé par la petite vérole ne m'a pas inspiré grand-chose, même si je comprends tout à fait l'intérêt historique pour des spécialistes. Enfin, je n' ai pas vraiment entendu parler de controverse à ce sujet, même si j'imagine que la finalité d'une telle reconstitution a pu susciter des interrogations. J'ai notamment cru comprendre que la reconstitution avait donné l'image d'un homme malade et affaibli, ce qui a peut-être pu créer un certain débat ; pour être tout à fait franche, je parle là par hypothèse, n'étant pas au courant des détails.

Aurore Chéry était à l'école primaire lors du Bicentenaire.

J'ai été à la fois intriguée - ce n'est pas pour rien que je travaille sur le XVIII ${ }^{\mathrm{e}}$ siècle - par cet événement et toutes les commémorations qui l'entouraient. Je m'intéresse au XVIII ${ }^{\mathrm{e}}$ siècle depuis ce moment - mais j'ai eu l'impression d'être instrumentalisée, oui, et c'était particulièrement déplaisant. Disons que c'est parce que je me suis sentie instrumentalisée que j'ai voulu en savoir plus. Mais sinon, oui, je garde de cette période 
une aversion profonde pour tout ce qui relève des processus mémoriels. Je comprends que certains puissent en avoir besoin mais pour moi, personnellement, je le vis comme quelque chose d'extrêmement déplaisant. En tant qu'enfants, on ne jugeait pas nécessaire de nous expliquer ce qui se passait. On devait planter des arbres de la liberté, chanter, c'était un folklore qui n'avait pas de sens. Il fallait le faire juste parce qu'on nous disait que c'était bien de le faire. Après mes années-lycée, qui ne m'ont pas laissé de grands souvenirs non plus sur ce sujet, je me suis beaucoup plus épanouie intellectuellement grâce à mes trois années de classe prépa qui ont été bien plus fondamentales. Préparant une thèse sur la période immédiatement précédente, je ne suis pas en prise avec les informations récentes liées à la Révolution. Je n'ai pas envie de faire une histoire du XVIII ${ }^{\mathrm{e}}$ siècle téléologique, ce qui m'intéresse c'est de comprendre comment pensaient les gens qui n'avaient pas conscience que la Révolution allait arriver. Après, il est vrai que je côtoie plus d'historiens de la Révolution et des périodes contemporaines parce que les modernistes qui travaillent sur des sujets proches des miens m'apparaissent souvent trop conservateurs. J'ai eu connaissance de la vente des papiers de Robespierre et de l'hommage à la Convention grâce à mon réseau Facebook, au CVUH et au site de l'IHRF, mais pas par celui de la SER que je ne consulte pas ${ }^{10}$. Mais quand j'y ai vu les photos des sans-culottes costumés chantant dans la rue, j'ai trouvé ça ridicule. J'ai entendu parler de l'affaire de la tête de Robespierre lorsque l'article de Guillaume [Mazeau] a circulé sur Facebook. Mais je n'ai pas suivi le reste, ça ne m'intéressait pas. Ma première pensée c'était : "Tiens, Charlier [le médecin qui prétend avoir retrouvé la vraie tête d'Henri IV] a encore trouvé quelque chose pour faire parler de lui. » Voilà, je n'ai pas grand-chose à raconter sur le sujet.

Emmanuel Laurentin revient sur La Fabrique de l'Histoire.

Lorsque j'ai créé mon émission, j'étais depuis peu très intéressé par les questions d'historiographie. L'historiographie d'une question est essentielle à connaître, et même à comprendre intimement, avant de se lancer dans l'étude. Elle montre en effet qu'il faut déconstruire les récits en cours car d'autres se sont attachés à remonter le fil du temps pour composer le fameux « roman national », qui n'est donc pas nécessairement le roman de tout un chacun. Il est pourtant encore difficile à l'historien de dire « je », de faire le récit des parcours de vie des individus, tant l'histoire scolaire

(10) CVUH : Comité de vigilance sur les usages de l'histoire ; SER : Société des études robespierristes. 
a construit une autre mythologie, une « fabrique des héros ». J'ai été très inspiré par un ouvrage qui portait ce titre et avait été publié dans les mois qui ont précédé le lancement de La Fabrique ${ }^{11}$. C'est ainsi que le choix de cette expression «Fabrique de l'Histoire » s'est imposé. En effet, il s'agit d'une fabrique, d'une construction en quelque sort manuelle, pierre à pierre, qui peut sans cesse être déconstruite pour être reconstruite. Il me semble que l'historiographie de la Révolution française a tardé, plus que pour d'autres sujets, à être remise en question. Le Bicentenaire, les travaux de Furet et de ses élèves, ont apporté un souffle d'air qui a permis de sortir d'une histoire convenue, à la fois marxiste et inspirée par Michelet. Mais les histoires des individus obscurs font encore défaut pour cette période, à mon avis, mais je ne suis pas du tout spécialiste ${ }^{12}$ ! J'ai eu connaissance de la vente des papiers de Robespierre et de la journée d'hommage à la Convention nationale mais je ne me souviens plus comment - sans doute via le fil professionnel des dépêches d'agence. Même chose quant à la reconstitution de la tête de Robespierre, annoncée par l'AFP. Je n'ai pas mis en doute l'information, que j'ai même diffusée sur twitter avant de lire votre message et l'article de Guillaume Mazeau sur FaceBook ${ }^{13}$. J'avais fait confiance à la source, sans trop de poser de questions.

Bien que journaliste également, Pascal Guimier reconnaît :

Non, je n'ai pas suivi l'histoire de la vente aux enchères des papiers de Robespierre ni la journée d'hommage à la Convention nationale. Je m'intéresse à l'Histoire de la Révolution française et j'ai suivi les débats portant sur la compréhension et l'historiographie de la Terreur. Est-ce un objet froid, comme l' affirment les tenants d'une histoire conservatrice, voire réactionnaire ? Est-ce un objet chaud, au contraire, capable, plus de deux siècles après, d'être une référence pour nos actions politiques actuelles? La question n'a pas cessé d'être débattue, de Joseph de Maistre à François Furet et de Michelet à Soboul et Vovelle ! C'est un patrimoine commun à ne pas oublier. À mon avis, en ce qui concerne les révolutions arabes, le rythme des événements a pu donner l'impression de quelques similitudes avec la Révolution française mais la suite a montré toute la spécificité des pays concernés, à peine dégagés de leur histoire coloniale et très soumis aux

(11) Pierre CENTLIVRes et alii (dir.), La fabrique des héros, Paris, Les éditions de la MSH, 1999.

(12) J'avais fait le même constat en préparant avec Éric Saunier le numéro spécial des $A H R F$, «Vivre la Révolution française », n³73, juillet-septembre 2013.

(13) Sur cette question, j'avais immédiatement réagi sur twitter, sachant que les têtes des décapités de Thermidor avaient été inhumées sous de la chaux vive. 
contraintes internationales, ce qui n'était pas et ne pouvait pas être le cas au $\mathrm{XVIII}^{\mathrm{e}}$ siècle ! Enfin, l'histoire de la tête de Robespierre, je crois en avoir eu connaissance dans Le Figaro, avec photo à l'appui. Ensuite l' «info » a été reprise par les chaînes tout-info et au moins France 2 qui adore la science et l'histoire expliquées aux petits ${ }^{14}$. Cela m'a rappelé la prétendue tête d'Henri IV dont la technique d'embaumement ne correspondait pourtant pas à ce que l'on sait des pratiques alors en vigueur mais on a réellement envie d'y croire ! La reconstitution faciale de Max-la-guillotine, d'après un masque mortuaire à l'authenticité contestée, me semble-t-il, me fait doucettement rigoler. En outre, diagnostiquer je ne sais quelle maladie au nom évoquant un précédent locataire de l'Élysée a tout de la supercherie. Quand on voit ledit visage, grêlé façon Gravelotte, on découvre le portrait vraisemblable d'un certain Danton. Cocasse, non ? Bref, on est plus proche de Barnum que du musée Grévin... Science, science, que de crimes commis en ton nom ! Quand l'info est estampillée «science », le journaleux est prêt à tout gober. Un effet « Jurassic Park »? Si c'est possible en fiction, ça pourrait être réel. C'est Oscar Wilde qui serait content !

Avec Emmanuel Fureix, je reprends le fil de la discussion sur les révolutions arabes.

\section{Emmanuel Fureix}

La survenue de ces mouvements révolutionnaires là où on les attendait le moins a suscité de nouvelles réflexions sur les idées de révolution et de régimes d'historicité. Est réapparu le sentiment que les peuples pouvaient reprendre leur destin en main et que le futur pouvait ouvrir à nouveau un espace d'émancipation. J'ai suivi avec un grand intérêt les interventions plus ou moins médiatiques des spécialistes de la Révolution sur les allers-retours possibles entre passé et présent. L'observation à ciel ouvert des situations révolutionnaires n'autorise pas à y voir le retour de « $1789 »$ (ni d'ailleurs de « $1848 »)$, encore moins de craindre une nouvelle «Terreur » ou de se projeter sur un nouveau « Brumaire ». Mais elle permet au contraire de renouveler les questions sur le passé des révolutions - par exemple sur le rôle du religieux et du sacré, ou sur l'imaginaire du futur dans les révolutions.

L'entretien avec Emmanuel Fureix se poursuit sur ce qui, dans les travaux récents, a renouvelé son regard sur la Révolution française :

(14) Dans les quelques jours suivants, toutes les chaînes ont répercuté l'info, de la même manière. Puis, elles ont inséré une brève interview de Guillaume Mazeau au Musée Carnavalet. 
La déconstruction de la Terreur, qui a été établie comme objet historiographique depuis le Directoire, et n'a cessé depuis d'être examinée à la lumière des connaissances. Or la multiplicité et la complexité des événements et des parcours individuels durant cette période nous a conduits à envisager aussi la période de la Terreur comme le champ de l'éclosion de nouveaux possibles. Mais c'est la force des anathèmes thermidoriens et directoriens que d'avoir figé un imaginaire de la Terreur jusque dans les années 1820 au moins. Ces images-écrans qui se sont succédé depuis 1795 ont obscurci les raisonnements. Il a fallu - par analogie avec le printemps 2011 - une nouvelle révolution, celle de 1830, et l'irruption de la question sociale pour que soit assumée à haute voix une autre lecture de la Terreur, autour de Buonarroti, de Laponneraye ou de Cabet, dont l'Histoire populaire de la Révolution française (1839-1840) forme un important jalon. Plus largement, «le renouveau » des regards sur la violence révolutionnaire est intéressant, tant du côté des analyses en termes d'équilibres politiques que de celui de la sacralité des droits. Il incite à lire avec circonspection les interprétations culturalistes des violences propres aux révolutions arabes. Le renouveau du regard porté sur les Antilles et la « révolution atlantique » est également impressionnant pour le dixneuvièmiste que je suis. Le développement d'une histoire transnationale ou connectée, à propos de la Révolution française comme des révolutions du $\mathrm{XIX}^{\mathrm{e}}$ siècle, aide à penser autrement des objets censés connus, il y a là un rapprochement supplémentaire entre nos deux périodes. Le colloque récent, en juin 2013 à Marne-La-Vallée, sur les « circulations révolutionnaires », en est l'illustration.

Enfin, sur une actualité plus factuelle à propos de Robespierre, il réagit ainsi :

Oui, bien sûr, j'ai suivi l'affaire de la vente aux enchères des papiers de Robespierre et la cérémonie d'hommage à la Convention. À propos de la reconstitution en $3 \mathrm{D}$ de la tête de Robespierre, de mémoire, j'ai eu vent de la nouvelle au JT en décembre dernier. J'ai suivi (d'un peu loin) la polémique née de l'affaire, notamment via le site du CVUH (le billet de Guillaume Mazeau avec qui j'en ai parlé depuis d'ailleurs), sans parler du débat public plus général, voire des discussions de comptoir! Ce que j'en ai pensé : c'est très instructif sur la force fantasmatique du personnage, à la hauteur d'Henri IV mais en symétrique négatif (la vérole contre la barbe fleurie !); instructif aussi sur la fascination scientiste pour la reconstitution des corps (3D - version moderne des masques mortuaires et autres reliques sentimentales des $\mathrm{XVIII}^{\mathrm{e}}$ et $\mathrm{XIX}^{\mathrm{e}}$ siècles); instructif 
également sur le choc de la parure (la perruque) et de la peau grêlée, qui participe lui-même au fantasme et au renouvellement du mythe. Mais on peut s'interroger sur la séparation étanche entre les compétences des uns et des autres (les historiens ne semblent pas avoir été sollicités, ni sur l'authenticité du masque et du récit de Mme Tussaud, alors qu'une bibliographie existe depuis longtemps, ni sur la question de la blessure à la mâchoire, etc) ${ }^{15}$. Cette histoire en dit long sur l'éclatement des savoirs, de plus en plus pointus mais aveugles entre eux.

Je commence mon entretien historiographique avec Pierre Serna en l'interrogeant sur l'une de ses déclarations récentes « Le temps des géants est passé. »

\section{Pierre Serna}

Effectivement, le problème de la Révolution française aujourd'hui est qu'elle est devenue un sujet d'histoire, peut-être le symptôme de quelque chose d'autre qu'elle-même, mais le temps de l'Histoire, lui, n'est pas passé. Cependant, elle vient à nous quand même, sans l'avoir demandée. Par exemple, le livre Pour quoi faire de la Révolution publiée sur la sollicitation du CVUH est à destination des jeunes collègues. L'équipe des cinq membres de l'IHRF qui ont cherché à montrer quels étaient les enjeux pour aujourd'hui de la recherche en histoire de la Révolution. Nous avons présenté ce livre dans beaucoup d'endroits : nous cherchons véritablement à médiatiser la recherche, le plus possible, mais par les bons créneaux. Médiatiser ne veut pas dire renoncer à la qualité intellectuelle, à l'érudition. L'expertise AERES de janvier 2013 a salué la qualité du site de l'Institut et la nouvelle revue électronique La Révolution française, sur le site Hypothèses.org, dont le numéro 5 portant sur le républicanisme anglais dans la France des Lumières et de la Révolution vient de paraître. Cette reconnaissance institutionnelle est importante car elle permet d'avoir des subventions : j'ai pu ainsi recruter un ingénieur d'études spécialisé dans les nouvelles technologies et une ingénieure d'études supplémentaire pour la publication « infinie » des Archives Parlementaires ${ }^{16}$. Tout cela demeure à confirmer dans le long terme et la volonté du CNRS de nous soutenir comme par le passé. En tant que directeur de l'IHRF mais aussi membre de

(15) Hector FlEISCHMANN, Le masque mortuaire de Robespierre, documents nouveaux pour servir d'intelligence et de conclusion à une polémique historique, Paris, Leroux, 1911.

(16) J'ai rencontré Toby Frajerman qui m'a expliqué sa politique de réseaux et de mise en ligne (refonte du site de l'Institut, amélioration du compte Facebook, développement de la newsletter...). 
la Société des études robespierristes, j'ai pris une part active dans l'achat des papiers de Robespierre et la célébration de la Convention ${ }^{17}$. Quant à « l'invention » du visage de Robespierre, je me suis tenu au courant comme tout un chacun par Internet, le site Facebook de l'IHRF et les envois du CVUH. Ce qui m'a frappé le plus... dans ce visage à la Danton ou à la Mirabeau... c'est, alors que pendant deux cents ans l'on a présenté la délicatesse gracile et juvénile du visage de Robespierre qu'une forme en tête de chat devait symboliser, non sans arrière-pensée, tant et si bien qu'un Dominique Jamet faisait semblant de dévoiler dans son roman médiocre en 1989 la prétendue relation de Robespierre et Saint-Just, voilà que ce Robespierre nous est présenté avec une face d'une masculinité à ce point affirmée qu'elle ne peut qu'interroger ${ }^{18}$. Quel est le sens de cette virilité outrée, de cette construction si genrée qu'elle donne un " mauvais genre », si l'expression est permise, à l'Incorruptible, ce que n'ont pas manqué de remarquer de nombreux observateurs, sans commenter pour autant ce côté hyper masculin? Je ne sais répondre à cette question.

\section{La question de la Vendée}

Concernant la télévision, l'épisode qui a récemment suscité la bronca des historiens de la Révolution française concerne le téléfilm Robespierre, bourreau de la Vendée, diffusé par la chaîne publique France 3 le 7 mars 2012 et à nouveau diffusé à plusieurs reprises depuis cette date, ce qui ne laisse pas de surprendre ${ }^{19}$. Les réactions des historiens professionnels ont été immédiates, de Marc Bélissa sur le site http://revolution-francaise.net/à Pierre Serna. «J'ai bien sûr réagi lorsque j'ai vu cette émission » confirme Pierre Serna qui rédige une lettre très argumentée pour le site de l'IHRF ${ }^{20}$.

\section{Pierre Serna}

J'ai choisi un titre assez choc mais qui correspond tout à fait à mon état d'esprit du moment Les trafiquants de mémoires ou la Vendée vendue....

(17) Voir dans le numéro spécial des $A H R F$ consacré à Robespierre, n³71, janvier-mars 2013, l'article de Serge ABERDAM et Cyril TRIOLAIRE, « La souscription nationale pour sauvegarder les manuscrits de Robespierre : introspection historique d'une initiative citoyenne et militante », p. 9-38.

(18) Dominique JAMET, Antoine et Maximilien, ou la Terreur sans la vertu, Paris, Denoël, 1986.

(19) Diffusion en première partie de soirée les 7 mars 2012 et 23 janvier 2013 et deux fois encore en deuxième partie de soirée, dont le 4 février 2013. Pierre Serna réagit à cette dernière diffusion. Désormais, l'intégralité du téléfilm peut être visionné sur YouTube.

(20) https://ihrf.univ-paris1.fr/centre-de-documentation/controverses/les-trafiquants-dememoires-ou-la-vendee-vendue/ 
Non spécialiste de la Vendée, et plutôt rétif, à tort, à la culture télévisuelle, j'avais décidé d'ignorer l'émission. Mais, devant ce qu'il convient d'appeler un acharnement médiatique dont le but ou la conséquence est de créer une vulgate partagée et finalement acceptée à force d'être répétée et ânonnée, quelques réflexions s'imposent. Un génocide aurait eu lieu en Vendée et qui dit génocide dit forcément que le coupable de l'histoire ou celui que l'on peut comparer à Hitler n'est personne d'autre que Robespierre. L'écriture télévisuelle, images et sons, joue sur les ressorts de l'émotion, de l'angoisse, pour enfermer le spectateur dans une sympathie à sens unique. Sans reprendre les approximations historiques, les facilités de vocabulaire (la Convention aurait affublé les Vendéens du nouveau qualificatif de «brigands » alors que c'était le terme justement employé par les cours de justice d'Ancien Régime contre tout acte rébellionnaire il m'a fallu donner des informations à tous ceux que ce téléfilm, et d'autres après lui, intéresse. On a remarqué que de nombreuses personnalités, choisies pour leurs compétences dans ce domaine, étaient de plus en plus souvent conviées à donner leur expertise, sans doute pour accorder du crédit à la réalisation. Si les propos de Jean-Clément Martin, spécialiste incontesté des guerres de Vendée, ont été conservés, quoique tronqués, que dire de ceux de Bernard Gainot ? Un long entretien lui a été demandé en tant que spécialiste des guerres révolutionnaires. Mais tous ses propos ont disparu (censure ?) au montage. Il avait osé comparer et montrer des parallèles entre la violence extrême des soldats bleus et la brutalité sans limite des soldats anglais au même moment contre les patriotes irlandais, ce qui inquiétait leurs officiers selon les témoignages de l'époque. Sûrement ces rappels historiques ne cadraient-ils pas avec la volonté délibérée de démontrer l'unicité du crime franco-français, hors de tout contexte de guerre... En revanche, Stéphane Courtois, le premier historien à intervenir dans l'émission et l'un des derniers à donner son avis à la fin, peut affirmer que «En France, il est impossible de critiquer la Révolution! ». Des journalistes courageux vont donc défaire le mythe à la Courtois pour faire la lumière sur le génocide vendéen... ou pas ${ }^{21}$ ! La seconde posture qui encadre le film est celle de Reynald Sécher qui soutient sans hésiter l'idée que l'on tue deux fois la Vendée par l'opération du mémoricide. Selon le polygraphe, on tue une seconde fois la Vendée en l'oubliant systématiquement, en refusant d'en parler, en l'omettant sciemment des histoires. Jean-Clément Martin peut

(21) On se rappelle que ce mot de « génocide » tout comme le décompte des victimes avait occupé les historiens dans les années pré-Bicentenaire. On est sidérés de voir revenir cette question en 2013. 
attester, par ses travaux et par les multiples sollicitations auxquelles il répond favorablement, qu'il n'en est rien $^{22}$. La méthode est celle qu'avait dénoncée Pierre Vidal-Naquet à propos des chambres à gaz : construire une fausseté que personne n'a jamais soutenue, pour, en la déconstruisant, remettre en doute une autre vérité qui, elle, a été établie ${ }^{23}$. Que font d'autre Stéphane Courtois et Reynald Sécher que d'utiliser ce procédé pour mettre en doute la valeur de la Révolution et instiller l'idée d'une volonté politique et planifiée de destruction d'une population ?

Parmi mes interlocuteurs, ni Cécile Guérin, ni Roland Timsit, ni Pascal Guimier, ni Emmanuel Laurentin n'ont vu l'émission. Ce dernier connaît la polémique car il a invité à plusieurs reprises les principaux chercheurs sur le sujet.

\section{Emmanuel Laurentin}

Il y a des tribunes, des prises de parole, des interviews qui montrent que le sujet est chaud chez les historiens et va au-delà du seul débat avec Reynald Sécher, Stéphane Courtois ou Alain Gérard ${ }^{24}$. On trouve encore sur Internet beaucoup de ces conférences qui font une sorte de ping-pong particulièrement entre Jean-Clément Martin et Sophie Wahnich d'ailleurs.

Interrogée sur le téléfilm Robespierre, bourreau de la Vendée? Aurore Chéry confie :

Je ne l'ai pas vu. Je ne m'intéresse pas à ces polémiques et je pense que le scénario comme les multidiffusions sont sans doute le fait d'un choix politico-culturel.

\section{Emmanuel Fureix relève :}

Nous sommes dans le registre de l'histoire des émotions, de l'histoire des larmes et des culpabilisations. Je n'ai pas vu le téléfilm mais l'histoire de la guerre de Vendée me paraît extrêmement propice à une récupération politique et à une tentative de confiscation de la mémoire. Il est intéressant de noter que c'est un député de la droite populaire, Lionnel Luca, qui a

(22) Lors du séminaire doctoral de l'IHRF du mercredi 29 janvier 2014 on a insisté sur le fait qu'entretenir l'idée de "mémoricide », contre toute évidence, vient des plus anti-républicains, réactionnaires et ultra-catholiques. Chaque année, députés et/ou sénateurs présentent une proposition de loi de « reconnaissance du génocide vendéen ». En 2010, elle a recueilli 22 députés, en 2012, 51 sénateurs. Mais bien peu de Vendéens parmi eux !

(23) Pierre VIDAL NAQUET, Les Assassins de la mémoire, "Un Eichmann de papier » et autres essais sur le révisionnisme, 1981, dernière édition augmentée, 2005, Paris Maspéro.

(24) Alain Gérard, chercheur associé à l'Université Paris IV, a été responsable du centre vendéen de recherches historiques jusqu'en août 2013. 
présenté la dernière proposition de loi mémorielle visant à reconnaître un génocide en Vendée. Le Journal officiel garde la trace de ses collègues : Véronique Besse, Jacques Bompard, Alain Leboeuf, Marion Maréchal-Le Pen, Alain Marleix et Yannick Moreau, ce dernier (UMP) étant le seul élu de Vendée ${ }^{25}$. C'est un marronnier législatif que Lionnel Luca entretient puisqu'il avait déjà présenté un texte en ce sens en 2007, sans guère d'écho à l'époque.

Pour conclure sur ce sujet, il faut mentionner aussi que des sites aussi différents idéologiquement que celui de Causeur ou celui de l'Humanité accueillent volontiers des personnalités pour exposer leurs idées et opinions sur la Révolution française. Serait-elle donc un objet chaud ? Chacun jugera. Malgré la présence d'affiches nombreuses un peu partout dans Paris pour promouvoir une comédie musicale, 1789. Les amants de la Bastille, aucun de mes interlocuteurs ne les avait remarquées ni, a fortiori, n'avait fait un lien avec notre sujet ${ }^{26}$. La Révolution française n'est pas près de disparaître de l'environnement médiatique, si l'on en juge par la récente initiative du domaine de Versailles de collaborer avec La Fabrique de l'Histoire et de consacrer une émission mensuelle à l'imaginaire historique de nos députés,

(25) http://www.assemblee-nationale.fr/14/propositions/pion0607.asp

(26) 1789. Les amants de la Bastille est un spectacle musical dont la première s'est déroulée le 10 octobre 2012. Il s'agit d'une fiction complète, une histoire d'amour se déroulant sur fond de Révolution française. 
les enregistrements devant se dérouler dans la salle du Jeu de Paume à Versailles !

Annie DUPRAT Université de Cergy-Pontoise CICC EA 2529 Annie.duprat@orange.fr

Cécile GuÉRIN cecile.guerin91@ orange.fr

Aurore CHERY aurore.chery@orange.fr

Pascal GUIMIER pascal.guimier@tv5monde.org

Pierre SERNA pierreserna@wanadoo.fr Roland TIMSIT rolandtimsit@gmail.com Emmanuel LAURENTIN emmanuellaurentin@free.fr Emmanuel FUREIX Efurex@free.fr 